\title{
New Solutions of the Einstein-Maxwell Equations from Old
}

\author{
B. Kent Harrison
}

Follow this and additional works at: https://scholarsarchive.byu.edu/facpub

Part of the Astrophysics and Astronomy Commons, and the Physics Commons

\section{Original Publication Citation}

Harrison, Kent B."New Solutions of the Einstein-Maxwell Equations from Old." Journal of Mathematical Physics 9 (1968): 1744-1752.

\section{BYU ScholarsArchive Citation}

Harrison, B. Kent, "New Solutions of the Einstein-Maxwell Equations from Old" (1968). Faculty Publications. 801.

https://scholarsarchive.byu.edu/facpub/801 
open map on $t_{P}$. Let $V$ be an arbitrary open subset of $t_{P}$, and let $V_{\alpha}$ be a covering of $t_{P}$ by open sets on each of which "exp" is an open map. For each $\alpha$, $V \cap V_{\alpha}$ is open in $V_{\alpha}$, and therefore $\exp \left(V \cap V_{\alpha}\right)$ is open in $M$. It follows that

$$
\exp (V)=\bigcup_{\alpha} \exp \left(V \cap V_{\alpha}\right)
$$

is open in $M$. Since $V$ is arbitrary, "exp" is an open map on $t_{P}$. Define $S_{P} \equiv \exp \left(t_{P}\right) \subset M$.

Now let $v$ be a tetrad at $P$. The tetrad $v$ determines a natural diffeomorphism $\varphi_{v}: T_{P} \rightarrow R^{4}$, where, for each vector $\xi \in T_{P}, \varphi_{v}(\xi)$ is defined as the 4-tuple of components of $\xi$ relative to $v$. We define the composition $\theta \equiv \exp \circ \varphi_{v}^{-1}$, a continuous map from a subset of $R^{4}$ into $M$

Whenever the integer $i$ is such that $q_{i} \in \theta^{-1}\left(S_{P}\right)$, we define

$$
\begin{aligned}
& P_{i}=\theta\left(q_{i}\right) \in M, \\
& U_{i} \equiv \theta\left(O_{i} \cap \theta^{-1}\left(S_{P}\right)\right) \subset M .
\end{aligned}
$$

At each of the points $P_{i}$ we define a tetrad $v_{i}$ by parallel transport of the tetrad $v$ along the geodesic ${ }^{37}$ from $P$ to $P_{i}$.

We now show that the $U_{i}$ are a basis for the open sets of $S_{p}$. Since the $O_{i}$ cover $R^{4}$, the $U_{i}$ cover $S_{P}$. Since $\theta$ is an open map on $\theta^{-1}\left(S_{P}\right)$, each $U_{i}$ is an open subset of $M$. Let $U$ be any open subset of $S_{P}$. Then $\theta^{-1}(U)$ is open in $R^{4}$. Since the $O_{i}$ are a basis for $R^{4}$,

${ }^{37}$ In case there are several geodesics from $P$ to $P_{i}$, let us select, for definiteness, that one which has been used (in the exponential map) to define $P_{i}$ we may select a collection $\Gamma$ of integers such that $\bigcup_{i \in \Gamma} O_{i}=\theta^{-1}(U)$. Therefore, $\bigcup_{i \in \Gamma} U_{i}=U$. Since $U$ is arbitrary, the $U_{i}$ are a basis for $S_{P}$.

To summarize, given any pair $(P, v)$, where $v$ is a tetrad at the point $P$, we have defined an open neighborhood $S_{P}$ of $P$ in $M$, a countable basis $U_{i}$ for $S_{P}$, a countable dense set $P_{i}$ in $S_{P}$, and a tetrad $v_{i}$ at each $P_{i}$. We now repeat our construction for each pair $\left(P_{i}, v_{i}\right)$. That is, we define an open neighborhood $S_{P_{i}}$ of $P_{i}$, a basis $U_{i j}$ for $S_{P_{i}}$, a dense set $P_{i j}$ in $S_{P_{i}}$, and a tetrad $v_{i j}$ at each $P_{i j}(i$ fixed, $j=1,2, \cdots)$. Applying the same construction to the pairs $\left(P_{i j}, v_{i j}\right)$, and so on, we obtain finally a countable collection $U_{\alpha} \equiv\left\{U_{i}\right.$, $\left.U_{i j}, U_{i j k}, \cdots\right\}$ of open sets of $M$ and a countable collection $P_{\alpha} \equiv\left\{P_{i}, P_{i j}, P_{i j k}, \cdots\right\}$ of points of $M$.

Since the $U_{\alpha}$ form a basis for each of the $S_{P_{\beta}}$, they also form a basis for $S \equiv \bigcup S_{P_{\alpha}}$. We must finally show that $S=M$.

Let $Q$ be any point in the closure of $S$. There is some neighborhood $U$ of $Q$ such that no geodesic segment in $U$ has a pair of conjugate points in $U$. Since $Q$ is in the closure of $S, U \cap S \neq \phi$. Therefore, since the $P_{\alpha}$ are dense in $S$, there must be some $P_{\beta} \in U$. But no geodesic segment has a pair of conjugate points in $U$, and so $S_{P_{\beta}} \supset U$. We have shown that $Q \in U \subset$ $S_{P_{\beta}} \subset S$, i.e., that $S$ contains each point of its closure. But $S$ is also open in $M$, because it is the union of the open sets $S_{P_{\alpha}}$. Since $M$ is connected, it follows that $S=M$. This completes the proof.

JOURNAL OF MATHEMATICAL PHYSICS VOLUME 9, NUMBER 11 NOVEMBER 1968

\title{
New Solutions of the Einstein-Maxwell Equations from Old*
}

\author{
B. KENT HARRISON $\dagger$ \\ Physics Department, Brigham Young University, Provo, Utah
}

(Received 14 December 1967)

\begin{abstract}
Methods are discussed with which one may derive theorems which allow one to generate new solutions of the Einstein-Maxwell equations from old ones. The old solutions used to generate new ones must admit at least one nonnull Killing vector and may be required to satisfy other conditions, depending on the theorem derived. Examples of derivable theorems are shown; these theorems are used in turn to show how generation of new solutions is accomplished. Examples of the latter are shown, such as generation of Brill or electrified NUT space from the Schwarzschild solution, generation of a new twisted Melvin universe from flat space, and generation of a new generalization of the Ozsvath-Schücking metric. Possible physical interpretations, uses, and extensions of this type of theorem are discussed.
\end{abstract}

\section{ASSUMED METRIC AND ITS EINSTEIN- MAXWELL EQUATIONS}

We outline a method, suitable for use on a wide class of metrics, by which one can derive theorems which in turn can be used to obtain new solutions of the Einstein-Maxwell equations from old. In Sec. 1, we find the form of the Einstein-Maxwell equations for the assumed class of metrics; in Sec. 2 we show how to derive these theorems. Section 3 presents examples of theorems, including one (Theorem 2) 
which is a generalization of and combination of previously derived theorems, one by Ehlers ${ }^{1}$ and one by the present author, ${ }^{2}$ Bonner, ${ }^{3}$ Papapetrou, ${ }^{4}$ Synge, ${ }^{5}$ and Misra and Radhakrishna. ${ }^{6}$ Section 4 gives examples of applications of these theorems to special metrics, and Sec. 5 discusses interpretations and uses.

We choose sign and other conventions as in Landau and Lifshitz. ${ }^{7}$ The assumed form of the metric is as follows:

$$
-d s^{2}=\epsilon\left[e^{2 U}\left(d x^{k}+a f_{\alpha} d x^{\alpha}\right)^{2}+a^{2} e^{-2 U} \gamma_{\alpha \beta} d x^{\alpha} d x^{\beta}\right],
$$

where

$$
\epsilon= \pm 1=\operatorname{sgn}\left(g_{k k}\right)
$$

$a$ is an arbitrarily chosen constant scale factor; $k$ is some particular one of $0,1,2,3$; Greek letters take all values of $0,1,2,3$, except $k$; and all metric coefficients are independent of $x^{k}$. Latin letters (except $k$ ) take on all values $0,1,2,3$. This metric thus admits a Killing vector $\xi^{l}=\delta_{k}^{l}$, but defines a congruence orthogonal to the hypersurfaces $x^{k}=$ const if $f_{\alpha}=0$.

We now define $\gamma^{\alpha \beta}$ as the inverse of the 3-dimensional metric $\gamma_{\alpha \delta}$,

$$
\gamma^{\alpha \beta} \gamma_{\alpha \delta}=\delta_{\delta}^{\beta}
$$

and $\sum_{\beta \gamma}^{\alpha}$ and $P_{\alpha \beta}$ as the Christoffel symbols and Ricci tensor, respectively, obtained from the $\gamma_{\alpha \beta}$. We also define

$$
h_{\alpha \beta}=f_{\alpha, \beta}-f_{\beta, \alpha},
$$

where the comma denotes ordinary differentiation, and we define the differential parameters of first and second order, ${ }^{8}$

$$
\begin{aligned}
\Delta_{1}(F) & =\gamma^{\alpha \beta} F_{, \alpha} F_{, \beta}, \\
\Delta_{1}(F, G) & =\gamma^{\alpha \beta} F_{, \alpha} G_{, \beta}, \\
\Delta_{2}(F)=\gamma^{\alpha \beta} F_{; \alpha \beta} & =\gamma^{\alpha \beta}\left(F_{, \alpha \beta}-\sum_{\alpha \beta}^{\gamma} F_{, \gamma}\right),
\end{aligned}
$$

* Part of this work was performed during the tenure of NSF Grant No. GP-4902; another part was performed while the author was a consultant at Jet Propulsion Laboratory, Pasadena, California. $\dagger$ Current (temporary) address: Jet Propulsion Laboratory, 4800 Oak Grove Drive, Pasadena, California 91103.

1 J. Ehlers, "Konstruktionen and Charakterisierungen von Lösungen der Einsteinschen Gravitationsfeldgleichungen" (dissertation, Hamburg, 1957).

${ }^{2}$ B. Kent Harrison, Phys. Rev. 138, B488 (1965); Report given at the London Conference on General Relativity, 1965.

3 W. B. Bonner, Z. Physik 161, 439 (1961).

4 A. Papapetrou, Proc. Roy. Irish Acad. A51, 191 (1947).

${ }^{5}$ J. L. Synge, Relativity: The General Theory (North-Holland Publishing Company, Amsterdam, 1960), pp. 367-71.

6 M. Misra and L. Radhakrishna, Proc. Nat. Inst. Sci. India A28, 632 (1962).

'L. Landau and E. Lifshitz, The Classical Theory of Fields (Addison-Wesley Publishing Company, Inc., Reading, Mass., 1962), 2nd ed., Chaps. 10 and 11.

${ }^{8}$ L. P. Eisenhart, Riemannian Geometry (Princeton University Press, Princeton, N.J., 1949), p. 41. where $F$ and $G$ are any functions of the coordinates. The semicolon always represents covariant differentiation with respect to the $\gamma_{\alpha \beta}$.

It is now a straightforward matter to calculate the components of the 4-dimensional Ricci tensor for metric (1). One obtains

$$
\begin{aligned}
R_{k k}= & a^{-2}\left[-e^{4 U} \Delta_{2}(U)+\frac{1}{4} e^{8 U} \gamma^{\alpha \beta} \gamma^{\gamma \delta} h_{\alpha \gamma} h_{\beta \delta}\right] \\
R_{k x}= & a f_{\alpha} R_{k k}+\frac{1}{2} a^{-1} e^{4 U} \gamma^{\delta \gamma}\left(-h_{\alpha \gamma, \delta}+\sum_{\delta \gamma}^{\epsilon} h_{\alpha \epsilon}\right. \\
& \left.+\sum_{\alpha \gamma}^{\epsilon} h_{\epsilon \delta}+4 h_{\delta \alpha} U_{, \gamma}\right), \\
R_{\alpha \beta}= & a f_{\alpha} R_{k \beta}+a f_{\beta} R_{k \alpha}-a^{2} f_{\alpha} f_{\beta} R_{k k}+P_{\alpha \beta} \\
& -2 U_{, \alpha} U_{, \beta}+\gamma_{\alpha \beta} \Delta_{2}(U)-\frac{1}{2} e^{4 U} \gamma^{\gamma \delta} h_{\alpha \gamma} h_{\beta \delta}
\end{aligned}
$$

We note from Eq. (4) that

$$
-h_{\alpha \gamma, \delta}=h_{\delta \alpha, \gamma}+h_{\gamma \delta, \alpha} .
$$

We thus may replace $-h_{\alpha \gamma, \delta}$ in Eq. (9) by $h_{\delta \alpha, \gamma}\left(h_{\gamma \delta, \alpha}\right.$ drops out because $h_{\gamma \delta}$ is antisymmetric and $\gamma^{\delta \gamma}$ is symmetric). Eq. (9) may then be written

$$
R_{k \alpha}=a f_{\alpha} R_{k k}+\frac{1}{2} a^{-1} e^{4 U} \gamma^{\delta \gamma}\left(h_{\delta \alpha, \gamma}+4 h_{\delta \alpha} U_{, \gamma}\right) \text {. }
$$

We choose units in the Einstein equations so that $G=c^{4}$. They thus may be written (for the vacuum or electromagnetic cases)

$$
R_{i j}=8 \pi T_{i j}
$$

where $T_{i j}$ is the energy-momentum tensor, since $T=0 . T_{i j}$ is defined by

$$
T_{i j}=(4 \pi)^{-1}\left(F_{i l} F_{j}^{l}-\frac{1}{4} F_{l m} F^{l m} g_{i j}\right) .
$$

Maxwell's equations are, for no sources,

$$
\begin{aligned}
& (-g)^{-\frac{1}{2}}\left[(-g)^{\frac{1}{2}} F^{i j}\right]_{, j}=0, \\
& F_{i j, l}+F_{l i, j}+F_{j l, i}=0 .
\end{aligned}
$$

We assume that the $F_{i j}$, also, are independent of $x^{k}$. The four Eqs. (14) then yield

$$
\begin{aligned}
& {\left[(-g)^{\frac{1}{2}} F^{k \alpha}\right]_{, \alpha}=0,} \\
& {\left[(-g)^{\frac{1}{2}} F^{\beta \alpha}\right]_{, \alpha}=0 .}
\end{aligned}
$$

Equations (15) may be written

$$
\begin{aligned}
F_{\alpha k, \beta}+F_{k \beta, \alpha} & =0, \\
\epsilon^{\alpha \beta \gamma} F_{\alpha \beta, \gamma} & =0,
\end{aligned}
$$

where $\epsilon^{\alpha \beta \gamma}$ is the alternating 3-index symbol $\left(\epsilon^{\alpha \beta \gamma}=+1\right.$ if $\alpha, \beta$, and $\gamma$ are in natural order). Equations (17) and (18) may be satisfied by choosing potentials $A$ and $B$ :

$$
\begin{aligned}
& F^{\alpha \beta}=(-g)^{-\frac{1}{2}} \epsilon^{\alpha \beta \gamma} A_{, \gamma}, \\
& F_{k \alpha}=B_{, \alpha} .
\end{aligned}
$$

Raising and lowering indices with the metric [Eq. (1)] 
now gives, after some algebra,

$$
\begin{aligned}
& F^{k \alpha}=a^{-2} \gamma^{\alpha \beta} B_{, \beta}+a(-g)^{-\frac{1}{2}} \epsilon^{\alpha \beta \gamma} f_{\beta} A_{, \gamma}, \\
& F_{\alpha \beta}=a\left(B_{, \beta} f_{\alpha}-B_{, \alpha} f_{\beta}\right)+a^{4}(-g)^{-\frac{1}{2}} e^{-4 U} \epsilon^{\gamma \delta \epsilon} \gamma_{\alpha \gamma} \gamma_{\beta \delta} A_{, \epsilon} .
\end{aligned}
$$

Substitution into Eqs. (16) and (19) yields the two remaining Maxwell equations:

$\Delta_{2}(A)-2 \Delta_{1}(U, A)+\frac{1}{2}(-\gamma)^{-\frac{1}{2}} e^{2 U} \epsilon^{\alpha \beta \gamma} h_{\alpha \beta} B_{, \gamma}=0$,

$\Delta_{2}(B)-2 \Delta_{1}(U, B)-\frac{1}{2}(-\gamma)^{-\frac{1}{2}} e^{2 U} \epsilon^{\alpha \beta \gamma} h_{\alpha \beta} A_{, \gamma}=0$,

where we have used Eq. (4) and

$$
g=a^{6} \gamma e^{-4 U} \text {. }
$$

We can now obtain the components of $T_{i j}$. We get

$$
\begin{aligned}
& T_{k k}=\epsilon\left(8 \pi a^{2}\right)^{-1} e^{2 U}\left[\Delta_{1}(A)+\Delta_{1}(B)\right], \\
& T_{k \alpha}=a f_{\alpha} T_{k k}+\epsilon(4 \pi a)^{-1}(-\gamma)^{-\frac{1}{2}} \epsilon^{\beta \gamma \delta} \gamma_{\alpha \beta} A_{, \delta} B_{, \gamma}, \\
& T_{\alpha \beta}=a f_{\alpha} T_{k \beta}+a f_{\beta} T_{k \alpha}-a^{2} f_{\alpha} f_{\beta} T_{k k} \\
& \quad+\epsilon(8 \pi)^{-1} e^{-2 U}\left\{2\left(A_{, \alpha} A_{, \beta}+B_{, \alpha} B_{, \beta}\right)\right. \\
& \left.\quad-\gamma_{\alpha \beta}\left[\Delta_{1}(A)+\Delta_{1}(B)\right]\right\} .
\end{aligned}
$$

Equations (12) now become, with Eqs. (8)-(10) and (27)-(29) and judicious algebraic combination,

$$
\begin{gathered}
\Delta_{2}(U)-\frac{1}{4} e^{4 U} \gamma^{\alpha \beta} \gamma^{\gamma \delta} h_{\alpha \gamma} h_{\beta \delta}=-\epsilon e^{-2 U}\left[\Delta_{1}(A)+\Delta_{1}(B)\right], \\
\gamma^{\delta \gamma}\left(h_{\delta \alpha ; \gamma}+4 h_{\delta \alpha} U_{, \gamma}\right)=4 \epsilon(-\gamma)^{-\frac{1}{2}} e^{-4 U} \epsilon^{\beta \gamma \delta} \gamma_{\alpha \beta} A_{, \delta} B_{, \gamma}, \\
P_{\alpha \beta}-2 U_{\alpha} U_{, \beta}+\frac{1}{4} e^{4 U} \gamma^{\gamma \delta}\left(\gamma_{\alpha \beta} \gamma^{\epsilon \eta} h_{\gamma \epsilon} h_{\delta \eta}-2 h_{\alpha \gamma} h_{\beta \delta}\right) \\
=2 \epsilon e^{-2 U}\left(A_{, \alpha} A_{, \beta}+B_{, \alpha} B_{, \beta}\right) .
\end{gathered}
$$

The equations we wish to solve are now Eqs. (4), (24), (25), (30), (31), and (32).

The quantities $h_{\alpha \beta}$ form a 3-dimensional antisymmetric tensor and can thus be expressed in terms of an axial vector. We write

$$
h_{\alpha \beta}=f_{\alpha, \beta}-f_{\beta, \alpha}=\epsilon_{\alpha \beta \gamma} \gamma^{\gamma \delta} z_{\delta}(-\gamma)^{\frac{1}{2}} .
$$

The integrability condition [Eq. (11)] becomes

$$
\gamma^{\alpha \beta} z_{\alpha ; \beta}=\gamma^{\alpha \beta}\left(z_{\alpha, \beta}-z_{\gamma} \sum_{\alpha \beta}^{\gamma}\right)=0 .
$$

Equations (24) and (25) become

$$
\begin{aligned}
& \Delta_{2}(A)-2 \Delta_{1}(U, A)+e^{2 U} \gamma^{\alpha \beta} B_{, \alpha} z_{\beta}=0, \\
& \Delta_{2}(B)-2 \Delta_{1}(U, B)-e^{2 U} \gamma^{\alpha \beta} A_{,{ }^{\alpha}} z_{\beta}=0,
\end{aligned}
$$

and Eqs. (30) $-(32)$ become

$$
\begin{aligned}
& \Delta_{2}(U)+\frac{1}{2} e^{4 U} \gamma^{\alpha \beta} z_{\alpha} z_{\beta}=-\epsilon e^{-2 U}\left[\Delta_{1}(A)+\Delta_{1}(B)\right], \\
& \epsilon^{\gamma \alpha \beta}\left(z_{\alpha, \beta}+4 z_{\alpha} U_{, \beta}+4 \epsilon e^{-4 U} B_{, \alpha} A_{, \beta}\right)=0, \\
& P_{\alpha \beta}-2 U_{, \alpha} U_{, \beta}-\frac{1}{2} e^{4 U} z_{\alpha} z_{\beta}=2 \epsilon e^{-2 U}\left(A_{, \alpha} A_{, \beta}+B_{, \alpha} B_{, \beta}\right) \text {. }
\end{aligned}
$$

We now note that Eq. (38) may be satisfied identically by choosing a "twist" potential $\phi$ in the following way:

$$
z_{\alpha}=e^{-4 U}\left[\phi_{, \alpha}+2 \epsilon\left(B A_{, \alpha}-A B_{, \alpha}\right)\right] .
$$

Eqs. (34), (35), (36), (38), (39) now become, respectively,

$$
\begin{aligned}
& \Delta_{2}(\phi)-4 \Delta_{1}(U, \phi)+2 \epsilon B\left[\Delta_{2}(A)-4 \Delta_{1}(U, A)\right] \\
& -2 \epsilon A\left[\Delta_{2}(B)-4 \Delta_{1}(U, B)\right]=0, \\
& \Delta_{2}(A)-2 \Delta_{1}(U, A) \\
& +e^{-2 U}\left[\Delta_{1}(\phi, B)+2 \epsilon B \Delta_{1}(A, B)-2 \epsilon A \Delta_{1}(B)\right]=0, \\
& \Delta_{2}(B)-2 \Delta_{1}(U, B) \\
& -e^{-2 U}\left[\Delta_{1}(\phi, A)+2 \epsilon B \Delta_{1}(A)-2 \epsilon A \Delta_{1}(A, B)\right]=0, \\
& \Delta_{2}(U)+\epsilon e^{-2 U}\left[\Delta_{1}(A)+\Delta_{1}(B)\right] \\
& +\frac{1}{2} e^{-4 U_{1}}\left[\Delta_{1}(\phi)+4 \epsilon B \Delta_{1}(\phi, A)-4 \epsilon A \Delta_{1}(\phi, B)\right. \\
& \left.+4 B^{2} \Delta_{1}(A)-8 A B \Delta_{1}(A, B)+4 A^{2} \Delta_{1}(B)\right]=0 \text {, } \\
& P_{\alpha \beta}=2 U_{, \alpha} U_{, \beta}+2 \epsilon e^{-2 U}\left(A_{, \alpha} A_{, \beta}+B_{, \alpha} B_{, \beta}\right) \\
& +\frac{1}{2} e^{-4 U}\left[\phi_{, \alpha}+2 \epsilon\left(B A_{, \alpha}-A B_{, \alpha}\right)\right] \\
& \times\left[\phi_{, \beta}+2 \epsilon\left(B A_{, \beta}-A B_{, \beta}\right)\right] \text {. }
\end{aligned}
$$

The symmetrical occurrence of $A$ and $B$ suggests the introduction of "polar potentials" $R$ and $\theta$ :

$$
\begin{aligned}
& A=R \cos \theta, \\
& B=R \sin \theta .
\end{aligned}
$$

Equations (40)-(45) now become, after suitable combination of Eq. (41), (42), and (43):

$$
z_{\alpha}=e^{-4 U}\left(\phi_{, \alpha}-2 \epsilon R^{2} \theta_{, \alpha}\right),
$$

$\Delta_{2}(\phi)-4 \Delta_{1}(U, \phi)+4 \epsilon R^{2} \Delta_{1}(U, \theta)$

$$
+2 R e^{-2 U}\left[2 R^{2} \Delta_{1}(R, \theta)-\epsilon \Delta_{1}(\phi, R)\right]=0,
$$

$$
\Delta_{2}(R)-R \Delta_{1}(\theta)-2 \Delta_{1}(U, R)
$$

$$
+\operatorname{Re}^{-2 U}\left[\Delta_{1}(\phi, \theta)-2 \epsilon R^{2} \Delta_{1}(\theta)\right]=0,
$$

$R \Delta_{2}(\theta)+2 \Delta_{1}(R, \theta)-2 R \Delta_{1}(U, \theta)$

$$
-e^{-2 U}\left[\Delta_{1}(\phi, R)-2 \epsilon R^{2} \Delta_{1}(R, \theta)\right]=0,
$$

$\Delta_{2}(U)+\epsilon e^{-2 U}\left[\Delta_{1}(R)+R^{2} \Delta_{1}(\theta)\right]$

$$
+\frac{1}{2} e^{-4 U}\left[\Delta_{1}(\phi)-4 \epsilon R^{2} \Delta_{1}(\phi, \theta)+4 R^{4} \Delta_{1}(\theta)\right]=0 \text {, }
$$

$$
\begin{aligned}
P_{\alpha \beta}= & 2 U_{, \alpha} U_{, \beta}+2 \epsilon e^{-2 U_{(}}\left(R_{, \alpha} R_{, \beta}+R^{2} \theta_{, \alpha} \theta_{, \beta}\right) \\
& +\frac{1}{2} e^{-4 U}\left(\phi_{, \alpha}-2 \epsilon R^{2} \theta_{, \alpha}\right)\left(\phi_{, \beta}-2 \epsilon R^{2} \theta_{, \beta}\right) .
\end{aligned}
$$

If now we have a solution of Eqs. (49)-(53) for the $\gamma_{\alpha \beta}$ and the four functions $U, \phi, R, \theta$, then Eq. (48) gives $z_{\alpha}$ and Eq. (33) can then be solved for the $f_{\alpha}$. 
It will be noted that since Eq. (33) is an equation for curl $\mathbf{f}$, there will exist an arbitrary function of integration $\xi$, with $\mathbf{f}=\boldsymbol{\nabla} \xi+$ other terms. From the form of the metric (1) we see immediately that $\xi$ can be absorbed into $x^{k}$ by redefinition of $x^{k}$, so that we will usually drop $\xi$. In certain situations it may be useful to use $\xi$ to change the form of the remaining terms in $f_{\alpha} d x^{\alpha}$.

\section{METHOD OF DERIVATION OF THEOREMS FOR GENERATING NEW SOLUTIONS OF THE EINSTEIN-MAXWELL EQUATIONS FROM OLD}

We assume first that a solution of Eqs. (49)-(53) is already known. The majority of known solutions of the Einstein-Maxwell equations fall into the class of metrics of form (1) and hence provide known solutions of the equations.

After a known solution has been selected, we identify the quantities $\gamma_{\alpha \beta}, U, f_{\alpha}, \epsilon$, and $a$ (usually set $=1$ ) by inspection. The electromagnetic potentials $A$ and $B$ may be identified by integrating a subset of the Eqs. (20)-(23). $z_{\delta}$ may be found from Eq. (33), and then $\phi$ may be found from Eq. (40). Equations (46) and (47) give $R$ and $\theta$ if desired.

One sees that vacuum solutions yield $A=B=0$, or $R=0$; solutions admitting a hypersurface-orthogonal congruence have $z_{\delta}=0$; solutions with constant $g_{k k}$ have $U=$ const. Thus in many cases, one or more of the function $U, \phi, R$, and $\theta$ are constant. We assume that at least one of these four functions is not constant.

To obtain new solutions of the Einstein-Maxwell equations, we assume the metric to have the form (1), with new $U, a$, and $f_{\alpha}$-denoted by a bar-but with the same $\gamma_{\alpha \beta}$ :

$$
-d s^{2}=\epsilon\left[e^{2 \widetilde{V}}\left(d x^{k}+\bar{a} \tilde{f}_{\alpha} d x^{\alpha}\right)^{2}+\bar{a}^{2} e^{-2 \tilde{U}} \gamma_{\alpha \beta} d x^{\alpha} d x^{\beta}\right] .
$$

We also assume new electromagnetic potentials $\bar{A}$ and $\bar{B}$-or $\bar{R}$ and $\bar{\theta}$. The $\bar{f}_{\alpha}$ are related to a new twist potential $\bar{\phi}$ by Eqs. (33) and (40) with bars. We now assume that $\bar{U}, \bar{\phi}, \bar{R}$, and $\vec{\theta}$ are functions of those among $U, \phi, R$, and $\theta$ which are not constant and of no other quantities.

It is easily shown that, with this assumption, the differential parameters of the barred quantities are linear functions of the differential parameters of the unbarred ones. For example, if $\bar{U}=\vec{U}(U, \phi)$, then

$$
\Delta_{1}(\bar{U})=\bar{U}_{U}^{2} \Delta_{1}(U)+2 \bar{U}_{U} \bar{U}_{\phi} \Delta_{1}(U, \phi)+\bar{U}_{\phi}^{2} \Delta_{1}(\phi),
$$

where subscripts denote differentiations. We now write Eqs. (49)-(52), barred, and use the assumed functional dependence to express them in terms of the differential parameters of the unbarred functions; substitute for the $\Delta_{2}$ 's from the original Eqs. (49)(52); then equate the coefficients of each of the $\Delta_{1}$ 's in each equation to zero. We do this last step because we wish to avoid restricting the original solution by imposing restrictions on $U, \phi, R$, and $\theta$. In some cases, the $\Delta_{1}$ 's of the original solution may satisfy relations among themselves; if this happens, these relations can be imposed on the Eqs. (49)-(52) before setting coefficients equal to zero, provided that the relations involve only functions of the $U, \phi, R$, and $\theta$.

When the coefficients are set equal to zero, we obtain a set of differential equations for the $\bar{U}$, etc., as functions of the $U$, etc. We obtain further equations by treating Eq. (53) in a similar manner. Since the $\gamma_{\alpha \beta}$ are the same in both metrics, the $P_{\alpha \beta}$ are also, and one may set the right-hand sides of the old and new Eqs. (53) equal. One then substitutes $\bar{U}=\bar{U}(U \cdots)$, etc., into one side and equates coefficients of the $U_{, \alpha} U_{, \beta}$, etc., terms.

There are four barred functions. If the number of nonconstant unbarred functions is $n$, one sees easily that, in general, there are $\frac{5}{2} n(n+1)$ differential equations for the four dependent variables as functions of the $n$ independent variables. There always exists one solution-the identity. Whether or not there exist solutions which are not equivalent to the identity is not at present known in general, although such do exist in many situations. We expect some redundancy in these partial differential equations, by the nature of the general-relativity equations themselves.

If solutions are obtained which are not equivalent to the identity, then these functions $\bar{U}$, etc., provide a new solution of the Einstein-Maxwell equations. It is, of course, desirable to include in it all arbitrary constants in order to provide greatest generality.

One can approach this treatment from a slightly different direction. In this, one assumes that certain of the unbarred functions are nonconstant-without specifying the particular metric satisfying this condition-and then finds the $\bar{U}$, etc. Then, if a metric is provided which satisfies the given condition on the unbarred functions, one already has the form of the $\bar{U}$, etc., and can immediately write down the new metric. This approach is clearly a powerful way of generating theorems describing how to generate new solutions from old. There are thus 15 possible theorems (15 equals the total number of combinations of four functions in all groupings) although not all may produce new inequivalent solutions.

Another variation in approach is obtained by assuming, for the original metric, that some of the $U, \phi, R$, and $\theta$ are functions of other nonconstant 
ones. This clearly places a restriction on the original metric; but if it is satisfied, then the number of differential equations for the $\bar{U}$, etc., is reduced. This approach is probably equivalent to assuming that there exist relations between the $\Delta_{1}$ 's of the original solution (possibility mentioned above). There are a large number of possible theorems derivable in this manner.

\section{EXAMPLES OF THEOREMS}

A simple beginning example is provided by considering a vacuum space with $g^{k k}=\epsilon$. In this case we have $U=0, R=0$. Rewrite $\phi$ as $\psi$. Examination of Eqs. (49)-(53) soon shows that

$$
\begin{aligned}
\Delta_{2}(\psi) & =0, \\
\Delta_{1}(\psi) & =0, \\
P_{\alpha \beta} & =\frac{1}{2} \psi_{, \alpha} \psi, \beta
\end{aligned}
$$

We find a new metric by writing $\bar{U}, \bar{\phi}, \bar{R}$, and $\bar{\theta}$ as functions of $\psi$. For simplicity we now drop the bars. Since $\Delta_{1}(F)$ and $\Delta_{2}(F)$ (where $F$ is any of $U, \phi, R$, and $\theta)$ are linear functions of $\Delta_{1}(\psi)$ and $\Delta_{2}(\psi)$, we see immediately by Eqs. (55) and (56) that the $\Delta_{1}(F)$ and $\Delta_{2}(F)$ are all zero, and that Eqs. (49)-(52) are satisfied identically. When we equate the new Eq. (53) to the old, and divide by $\psi_{. \alpha} \psi_{, \beta}$, we get

$$
\begin{aligned}
\frac{1}{2}=2 U^{\prime 2}+2 \epsilon e^{-2 U}\left(R^{\prime 2}\right. & \left.+R^{2} \theta^{\prime 2}\right) \\
& +\frac{1}{2} e^{-4 U}\left(\phi^{\prime}-2 \epsilon R^{2} \theta^{\prime}\right)^{2},
\end{aligned}
$$

where a prime denotes $d / d \psi$. The identity transformation is the case $U=0, R=0$, and $\phi=\psi$.

Solving for $\phi^{\prime}$ gives

$\phi^{\prime}=2 \epsilon R^{2} \theta^{\prime} \pm e^{2 U}\left[1-4 U^{\prime 2}-2 \epsilon e^{-2 U}\left(R^{\prime 2}+R^{2} \theta^{\prime 2}\right)\right]^{\frac{1}{2}}$.

Thus, using the forms of the metrics (1) and (54) and Eqs. (33) and (48), we can write the following theorem:

Theorem 1: For every vacuum metric which is a solution of the Einstein equations, and of the form (with $\partial / \partial x^{k}=0$ )

$$
-d s^{2}=\epsilon\left[\left(d x^{k}+f_{\alpha} d x^{\alpha}\right)^{2}+\gamma_{\alpha \beta} d x^{\alpha} d x^{\beta}\right],
$$

we define $\psi$ by the equations

$$
f_{\alpha, \beta}-f_{\beta, \alpha}=\epsilon_{\alpha \beta \gamma} \gamma^{\gamma \delta} \psi, \delta(-\gamma)^{\frac{1}{2}}
$$

(we assume $\psi$ nonconstant). Then there exists a generalized solution, including electromagnetic fields, of the form

$$
-d s^{2}=\epsilon\left[e^{2 U}\left(d x^{k}+a \bar{f}_{\alpha} d x^{\alpha}\right)^{2}+a^{2} e^{-2 U} \gamma_{\alpha \beta} d x^{\alpha} d x^{\beta}\right],
$$

and with electromagnetic potentials $R, \theta$ such that $U, R, \theta$ are arbitrary functions of $\psi$, and with

$$
\begin{aligned}
\bar{f}_{\alpha, \beta}-\bar{f}_{\beta, \alpha} & =\epsilon_{\alpha \beta \gamma} \gamma^{\gamma \delta} \psi, \delta(-\gamma)^{\frac{1}{2}} G(\psi) \\
& =G(\psi)\left(f_{\alpha, \beta}-f_{\beta, \alpha}\right),
\end{aligned}
$$

where

$$
G(\psi)= \pm e^{-2 U}\left[1-4 U^{\prime 2}-2 \epsilon e^{-2 U}\left(R^{\prime 2}+R^{2} \theta^{\prime 2}\right)\right]^{\frac{1}{2}} .
$$

The expression $R^{\prime 2}+R^{2} \theta^{\prime 2}$ may be replaced by $A^{\prime 2}+B^{\prime 2}$.

For our second theorem, we work with the "rectangular" potentials $A$ and $B$. We first assume that $\phi$, $A$, and $B$ are functions of $U$; this constitutes a restriction on the original metric chosen. (These restrictions are satisfied automatically for a vacuum metric admitting trajectories orthogonal to hypersurfaces $x^{k}=$ const.)

We first find the form of the equations under this restriction. Equation (40) gives

$$
z_{\alpha}=e^{-4 U}\left[\phi^{\prime}+2 \epsilon\left(B A^{\prime}-A B^{\prime}\right)\right] U_{, a},
$$

which we write for simplicity as

$$
z_{\alpha}=H(U) U_{, \alpha},
$$

and we have

$$
\begin{aligned}
& A=A(U), \\
& B=B(U) .
\end{aligned}
$$

It is simplest to work with Eqs. (34)-(39); they become

$$
\begin{gathered}
H \Delta_{2}(U)+H^{\prime} \Delta_{1}(U)=0, \\
A^{\prime} \Delta_{2}(U)+\left(A^{\prime \prime}-2 A^{\prime}+H B^{\prime} e^{2 U}\right) \Delta_{1}(U)=0, \\
B^{\prime} \Delta_{2}(U)+\left(B^{\prime \prime}-2 B^{\prime}-H A^{\prime} e^{2 U}\right) \Delta_{1}(U)=0, \\
\Delta_{2}(U)+\left[\frac{1}{2} e^{4 U} H^{2}+\epsilon e^{-2 U}\left(A^{\prime 2}+B^{\prime 2}\right)\right] \Delta_{1}(U)=0, \\
P_{\alpha \beta}=\left[2+\frac{1}{2} H^{2} e^{4 U}+2 \epsilon e^{-2 U}\left(A^{\prime 2}+B^{\prime 2}\right)\right] U_{, \alpha} U_{, \beta},
\end{gathered}
$$

where a prime denotes $d / d U$. We use Eq. (70) to eliminate $\Delta_{2}(U)$ and assume $\Delta_{1}(U) \neq 0$; Eqs. (67)(69) become

$$
\begin{gathered}
H^{\prime}=G H, \\
A^{\prime \prime}-(G+2) A^{\prime}+H B^{\prime} e^{2 U}=0, \\
B^{\prime \prime}-(G+2) B^{\prime}-H A^{\prime} e^{2 U}=0,
\end{gathered}
$$

where

$$
G=\frac{1}{2} e^{4 U} H^{2}+\epsilon e^{-2 U}\left(A^{\prime 2}+B^{2}\right) .
$$


If we put

we find

$$
\begin{aligned}
& H=L e^{-2 U} \\
& A^{\prime}=Q e^{U} \cos \tau, \\
& B^{\prime}=Q e^{U} \sin \tau,
\end{aligned}
$$

Equation (71) becomes

$$
P_{\alpha \beta}=\left(2+\frac{1}{2} L^{2}+2 \epsilon Q^{2}\right) U_{, \alpha} U_{, \beta} .
$$

Solution of Eqs. (79)-(82) gives

$$
\begin{aligned}
Q^{2} & =\epsilon \lambda e^{2 U}\left(1-\lambda e^{2 U}-\mu e^{4 U}\right)^{-1}, \\
L^{2} & =4 \mu e^{4 U}\left(1-\lambda e^{2 U}-\mu e^{4 U}\right)^{-1}, \\
\tau & \left.=\int L d U+\nu \quad \text { (vacuous if } \lambda=0\right),
\end{aligned}
$$

where $\lambda, \mu$, and $\nu$ are arbitrary constants. If we consider separately the cases with $\lambda=0$ and $\lambda \neq 0$, and those with $\mu=0$ and $\mu \neq 0$, and if we perform the integrations indicated in Eqs. (77), (78), and (86), we find a general form of the remaining equations as follows [including Eq. (33)]:

$$
\begin{gathered}
f_{\alpha, \beta}-f_{\beta, \alpha} \\
=2 \omega \epsilon_{\alpha \beta \gamma} \gamma^{\gamma \delta}\left[\mu\left(1-\lambda e^{2 U}-\mu e^{4 U}\right)^{-1}\right]^{\frac{1}{2}}(-\gamma)^{\frac{1}{2}} U_{, \delta},
\end{gathered}
$$

where $\alpha, A_{0}, B_{0}$ are constants, $\omega= \pm 1, \delta= \pm 1$, and $\xi=\epsilon \lambda\left(\lambda^{2}+4 \mu\right)^{-1}$. If both $\lambda$ and $\mu=0, A$ and $B$ are to be taken as constants. Constants $A_{0}$ and $B_{0}$ are trivial, since they do not appear in the electromagnetic fields; the constant $\alpha$ signifies a duality rotation. It will be noted that Eqs. (84) and (85) require

$$
\epsilon \lambda \mu \geq 0
$$

and

$$
4 \mu+\lambda^{2} \geq\left(2 \mu e^{2 U}+\lambda\right)^{2} \geq 0 .
$$

These conditions insure that the quantities under the square roots above will be positive or zero.
We now assume a second solution of the same form as the first and denote all quantities by bars, as before. We now assume $\bar{U}=\bar{U}(U)$. Equation (88), old and new, yields

$$
\begin{aligned}
P_{\alpha \beta} & =2\left(1-\lambda e^{2 U}-\mu e^{4 U}\right)^{-1} U_{, \alpha} U_{, \beta} \\
& =2\left(1-\bar{\lambda} e^{2 \bar{U}}-\bar{\mu} e^{4 \bar{U}^{-1}} \bar{U}_{, \alpha} \bar{U}_{, \beta},\right.
\end{aligned}
$$

which gives, since $\bar{U}=\bar{U}(U)$,

$$
(d \bar{U} / d U)^{2}=\left(1-\bar{\lambda} e^{2 \bar{U}}-\bar{\mu} e^{4 \bar{U}}\right)\left(1-\lambda e^{2 U}-\mu e^{4 U}\right)^{-1} .
$$

Also

and

giving

$$
\Delta_{2}(\bar{U})=\bar{U}^{\prime \prime} \Delta_{1}(U)+\bar{U}^{\prime} \Delta_{2}(U)
$$

$$
\begin{aligned}
-\left(\bar{\lambda} e^{2 \bar{U}}+2 \bar{\mu} e^{4 \bar{U}}\right)\left(1-\bar{\lambda} e^{2 \bar{U}}-\bar{\mu} e^{4 \bar{U}}\right)^{-1} \bar{U}^{2} \Delta_{1}(U) \\
=\bar{U}^{\prime \prime} \Delta_{1}(U)-\bar{U}^{\prime}\left(\lambda e^{2 U}+2 \mu e^{4 U}\right) \\
\times\left(1-\lambda e^{2 U}-\mu e^{4 U}\right)^{-1} \Delta_{1}(U) .
\end{aligned}
$$

We divide by $\Delta_{1}(U)$ and obtain a second-order differential equation for $\bar{U}(U)$. Equation (94) is a first integral of Eq. (95), so we consider only Eq. (94).

To integrate Eq. (94), we write

$$
\begin{aligned}
& X=e^{-2 U}-\frac{1}{2} \lambda, \\
& \bar{X}=e^{-2 U^{\tau}}-\frac{1}{2} \bar{\lambda}, \\
& \beta^{2}=\mu+\frac{1}{4} \lambda^{2}, \\
& \bar{\beta}^{2}=\bar{\mu}+\frac{1}{4} \bar{\lambda}^{2} .
\end{aligned}
$$

Equation (94) then becomes

$$
(d \bar{X} / d X)^{2}=\left(\bar{X}^{2}-\bar{\beta}^{2}\right)\left(X^{2}-\beta^{2}\right)^{-1} .
$$

The solutions of Eq. (98) fall into a number of cases, depending on the values of $\beta$ and $\bar{\beta}$. We note, by Eq. (93), that if $\beta=0,2 \mu e^{2 U}+\lambda=0$, yielding $\mu=\lambda=0$.

If $\mu=0$, we have a metric with a congruence orthogonal to the hypersurfaces $x^{k}=$ const; such metrics will be denoted by the letter $O$. If $\lambda=0$, then $\xi=0$, and $A$ and $B$ reduce to constants, so that we have a vacuum space; we denote these spaces by $\mathrm{V}$. If $\lambda \neq 0$, we have electromagnetic fields presentdenoted by $E$. Thus we may classify solutions as in Table I.

TABLE I. Classification of solutions of the metric.

\begin{tabular}{cllll}
\hline Type & OV & OE & V & E \\
\hline$\lambda$ & $=0$ & $\neq 0$ & $=0$ & $\neq 0$ \\
$\mu$ & $=0$ & $=0$ & $\neq 0$ & $\neq 0$ \\
\hline
\end{tabular}


TABLE II. Solutions of Equation (98), listing the conditions which apply, and the classifications of the old and new solutions.

\begin{tabular}{|c|c|c|c|c|}
\hline Case & Conditions & $\bar{X}(X)$ & Old solution & New solution \\
\hline $\begin{array}{l}1 \\
2\end{array}$ & $\begin{array}{l}\beta=\bar{\beta}=0 \\
\beta=\bar{\beta}=0\end{array}$ & $\begin{array}{c}\bar{X}=k X \\
\bar{X}=k X^{-1}\end{array}$ & $\begin{array}{l}\text { OV } \\
\text { OV }\end{array}$ & $\begin{array}{l}\text { OV } \\
\text { OV }\end{array}$ \\
\hline 3 & $\beta \neq 0, \bar{\beta}=0$ & $\bar{X}=k \beta^{-1}\left(X \pm \sqrt{X^{2}-\beta^{2}}\right)$ & OE, V, E & ov \\
\hline 4 & $\beta=0, \bar{\beta} \neq 0$ & $\bar{X}=\bar{\beta}(2 k X)^{-1}\left(X^{2}+k^{2}\right)$ & OV & $\mathrm{OE}, \mathrm{V}, \mathrm{E}$ \\
\hline 5 & $\beta \bar{\beta} \neq 0, X^{2}>\beta^{2}$ & $\bar{X}=\bar{\beta} \beta^{-1}\left(X \cosh k+\sqrt{X^{2}-\beta^{2}} \sinh k\right)$ & OE, V, E & OE, V, E \\
\hline 6 & $\beta \bar{\beta} \neq 0, X^{2}<\beta^{2}$ & $X=\bar{\beta} \beta^{-1}\left(X \cos k+\sqrt{\beta^{2}-X^{2}} \sin k\right)$ & OE, V, E & $\mathrm{OE}, \mathrm{V}, \mathrm{E}$ \\
\hline
\end{tabular}

We now (Table II) list the various kinds of solutions of Eq. (98), listing the conditions which apply and the classifications of the old and new solutions. In all cases, $k$ is the constant of integration.

Case 1 is trivial; Case 2 very nearly so. Case 4 , with $\bar{\lambda}=0$, is the case treated by Ehlers ${ }^{1}$; Case 4 , with $\bar{\mu}=0$, is the one treated by the author and others. ${ }^{2-6}$

As noted before, OV metrics satisfy restrictions (65) and (66) automatically and thus can be used via Cases (2) and (4) above to generate new metrics. Metrics which are $O E, V$, or $E$ need to be checked via Eqs. (65), (66) to see if they can be used to generate new metrics.

Thus Theorem 2 can be stated as follows: If we have a metric of form (1), satisfying Eqs. (65) and (66), then we can write down new metrics of the same form by using one or more of the cases of Table II. As a practical procedure, we identify $\epsilon, U, f_{\alpha}, \gamma_{\alpha \beta}, A, B$ from the form (1) and the known electromagnetic fields [Eqs. (20)-(23)]. We then find $z_{\delta}$ from Eq. (33) and check Eqs. (65) and (66). If they are satisfied, we identify $\omega, \delta, \mu$, and $\lambda$ from Eqs. (87), (90), and (91). Equations (96a) and (97a) give $X$ and $\beta$; then Table II can guide us to a selection of cases and functions $X$. We then reverse the procedure, using Eqs. (96b), (87), (90), and (91) to get $U, \tilde{f}_{\alpha}, \bar{A}$, and $\bar{B}$. Equations (65) and (66) are now automatically satisfied. $k, \bar{\lambda}$, $\bar{\mu}$ are to be considered as arbitrary constants of integration.

\section{APPLICATIONS OF THEOREMS}

Three examples of the uses of these theorems are presented here. They are not discussed in detail since that is beyond the scope of this paper.

Example 1: We generalize the Ozsvath-Schücking metric ${ }^{9}$ by using Theorem 1. It is given by

$$
\begin{aligned}
-d s^{2}=\left(d x^{1}\right)^{2}-4 v d x^{1} d x^{3} & +2 d x^{2} d x^{3} \\
& +2 v^{2}\left(d x^{3}\right)^{2}+(d v)^{2},
\end{aligned}
$$

where $v=x^{4}$. We choose $x^{k}=x^{1}$ and then identify the

\footnotetext{
9 I. Ozsvath and E. Schücking, Recent Developments in General
} Relativity (Pergamon Press, New York, 1962), p. 339. following quantities: $\epsilon=1, a=1, U=0, A=$ $B=0, f_{0}=f_{2}=0, f_{3}=-2 v, \quad \gamma_{00}=1, \quad \gamma_{23}=1$, $\gamma_{33}=-2 v^{2}, \gamma_{22}=\gamma_{02}=\gamma_{03}=0, g=\gamma=-1$. Equation (33) gives $z_{0}=z_{2}=0, z_{3}=-2$, so that Eq. (40) (with $\phi \rightarrow \psi$ ) gives

$$
\psi=-2 x^{3}
$$

Integration of Eq. (63), dropping of arbitrary functions of integration, yields

$$
\bar{f}_{0}=\bar{f}_{2}=0, \quad \bar{f}_{3}=-2 v G(\psi) .
$$

We use Eq. (100) to express everything in terms of $x^{3}$. By Eq. (62) we get, finally, that

$$
\begin{aligned}
-d s^{2} & =e^{2 U}\left[d x^{1}-2 a v F\left(x^{3}\right) d x^{3}\right]^{2} \\
& +a^{2} e^{-2 U}\left[2 d x^{2} d x^{3}-2 v^{2}\left(d x^{3}\right)^{2}+(d v)^{2}\right]
\end{aligned}
$$

is a solution of the Einstein-Maxwell equations, where $a$ is an arbitrary constant, $U, A$, and $B$ are arbitrary functions of $x^{3}$,

$$
F\left(x^{3}\right)=e^{-2 U}\left[1-U_{, 3}^{2}-\frac{1}{2} e^{-2 U}\left(A_{, 3}^{2}+B_{, 3}^{2}\right)\right]^{\frac{1}{2}},
$$

and the subscript 3 indicates $d / d x^{3}$. $A$ and $B$ occur as electromagnetic potentials and yield the following electromagnetic fields:

$$
\begin{gathered}
F^{02}=a^{-3} e^{2 U} A_{, 3}, \quad F_{13}=B_{, 3}, \\
F^{12}=a^{-2} B_{, 3}, \quad F_{03}=a e^{-2 U} A_{, 3} .
\end{gathered}
$$

To obtain the locally observable electromagnetic fields $\mathbf{E}$ and $\mathbf{H}$, we write the metric in terms of differential 1 forms:

$$
-d s^{2}=\left(\omega^{1}\right)^{2}+\left(\omega^{2}\right)^{2}+\left(\omega^{3}\right)^{2}-\left(\omega^{0}\right)^{2},
$$

where

$$
\begin{aligned}
& \omega^{0}=a e^{-U}\left[\sqrt{2} v d x^{3}-(\sqrt{2} v)^{-1} d x^{2}\right] \\
& \omega^{1}=e^{U}\left(d x^{1}-2 a v F d x^{3}\right) \\
& \omega^{2}=a e^{-U}(\sqrt{2} v)^{-1} d x^{2} \\
& \omega^{3}=a e^{-U} d v
\end{aligned}
$$

and write the electromagnetic field 2-form

$$
\begin{aligned}
\phi= & \frac{1}{2} F_{i j} d x^{i} \wedge d x^{j}=\left(E_{1} \omega^{1}+E_{2} \omega^{2}+E_{3} \omega^{3}\right) \wedge \omega^{0} \\
& +H_{1} \omega^{2} \wedge \omega^{3}+H_{2} \omega^{3} \wedge \omega^{1}+H_{3} \omega^{1} \wedge \omega^{2} .
\end{aligned}
$$


We obtain, by using Eqs. (104), (106), and (107),

$$
\begin{aligned}
& E_{3}=-H_{1}=(\sqrt{2} v a)^{-1} A_{, 3}, \\
& E_{1}=H_{3}=(\sqrt{2} v a)^{-1} B_{, 3}, \\
& E_{2}=H_{2}=0 .
\end{aligned}
$$

This is clearly a plane wave, since $\mathbf{E} \cdot \mathbf{H}=\mathbf{0}$ and $\mathbf{E}^{2}-\mathbf{H}^{2}=0$ everywhere. Its direction of propagation is in the $x^{2}$ direction. This result is satisfying, since the original Oszvath-Schücking metric (and also, apparently, this new one) represents a gravitational plane wave traveling in the $x^{2}$ direction.

The above comments are somewhat faulty, since they are based on a tetrad [components of Eqs. (106)] which is singular at $v=x^{4}=0$. One can define a nonsingular tetrad for the above metric, in the manner of Oszvath-Schücking, ${ }^{9}$ but its form is quite complicated and it will not be given here. One does find that nonsingularity is preserved only if $\left(\mathrm{Fe}^{2 V}\right)^{2}>\frac{1}{2}$, which yields

$$
1>2 U_{, 3}^{2}+e^{-2 U}\left(A_{, 3}^{2}+B_{, 3}^{2}\right) .
$$

This expression bears some resemblance to terms in the energy-momentum tensor and tempts one to surmise that if the energy density gets too large, a singularity develops and gravitational collapse takes place, but this point remains to be investigated elsewhere, along with more detailed studies of the metric (102).

Example 2: We begin with a flat metric in cylindrical coordinates

$$
-d s^{2}=-d T^{2}+d r^{2}+r^{2} d \theta^{2}+d z^{2}
$$

and use Theorem 2, Case 4. We choose $x^{k}=\theta\left(=x^{2}\right)$ and note that $\epsilon=1, a=1, f_{\alpha}=0, U=\ln r$, $-\gamma_{00}=\gamma_{11}=\gamma_{33}=r^{2}, \quad \gamma_{01}=\gamma_{03}=\gamma_{13}=0, \quad \gamma=$ $-r^{6}$. Thus $\lambda=\mu=0, \beta=0$. If we put $\tilde{\beta}=2 k l^{2}$, $\bar{\lambda}=4 c k l^{2}$, we find

$$
\begin{gathered}
e^{-2 C}=l^{2} r^{-2}\left(k^{2} r^{4}+2 c k r^{2}+1\right), \\
\tilde{f}_{1}=\tilde{f}_{3}=0, \quad \tilde{f}_{0}=4 k e^{2} \sqrt{1-c^{2}} z,
\end{gathered}
$$

and

$$
\begin{aligned}
& C=l^{-1} \sqrt{k c\left(1-c^{2}\right)} r^{2}\left(k^{2} r^{4}+2 c k r^{2}+1\right)^{-1}, \quad(113) \\
& D= \pm(2 l)^{-1} \sqrt{c k^{-1}}\left(1-k^{2} r^{4}\right)\left(k^{2} r^{4}+2 c k r^{2}+1\right)^{-1},
\end{aligned}
$$

where

$$
\begin{aligned}
& \bar{A}=C \cos \alpha+D \sin \alpha, \\
& \bar{B}=C \sin \alpha-D \cos \alpha
\end{aligned}
$$

( $\alpha$ represents a duality rotation).
The locally observed electromagnetic fields are both in the $z$ direction and are given by

$$
\begin{aligned}
& E=\zeta \cos \alpha+\gamma \sin \alpha, \\
& H=-\zeta \sin \alpha+\gamma \cos \alpha,
\end{aligned}
$$

with

$$
\begin{aligned}
& \zeta=(a r)^{-1} d C / d r, \\
& \gamma=(a r)^{-1} d D / d r .
\end{aligned}
$$

Inspection shows that this is a "twisted" Melvin magnetic universe. The twist is given by $\bar{f}_{0}$, which plays the role of an angular velocity in a rotating coordinate system. Since $\tilde{f}_{0}$ is proportional to $z$, we see that this angular velocity increases as $z$ increases and is of opposite signs on the two sides of $z=0$. One envisions a universe twisting upon itself (or untwisting!) as time goes on.

The case $c= \pm 1$ yields the usual Melvin universe, ${ }^{10}$ with no twist. The case $c=0$ gives maximum twist, but no electromagnetic field. Thus the twist and the electromagnetic field are complementary in some sense.

It is easily shown that a twisted Melvin universe, singular at $r=0$ and reducing to the ordinary singular Melvin universe ${ }^{10}$ for $c= \pm 1$ as above, can be obtained from the Weyl-Levi-Civita metric for a line mass.

Example 3: We use Theorem 2, Part 4 again, to generate a generalization of the Schwarzschild solution. We choose $x^{k}=x^{0}=T$, with $a=1, \epsilon=-1$, $f_{\alpha}=0, \quad U=\frac{1}{2} \ln (1-2 M / R), \quad \gamma_{R R}=-1, \quad \gamma_{\theta \theta}=$ $-R^{2}(1-2 M / R), \gamma_{\phi \phi}=-R^{2}(1-2 M / R) \sin ^{2} \theta, \gamma_{\alpha \beta}=$ $0(\alpha \neq \beta)$. With $\bar{\beta}=2 k l^{2}$ and $\bar{\lambda}=4 c k l^{2}$ as before, we get

$$
\begin{aligned}
& e^{-2 \widetilde{U}}=l^{2}\left[(1-2 M / R)^{-1}+k^{2}(1-2 M / R)+2 c k\right], \\
& f_{1}=f_{2}=0, f_{3}=4 k l^{2}\left(1-c^{2}\right)^{\frac{1}{2}} M \cos \theta, \\
& C=l^{-1}\left[-c k\left(1-c^{2}\right)\right]^{\frac{1}{2}}\left[(1-2 M / R)^{-1}\right. \\
& \left.+k^{2}(1-2 M / R)+2 c k\right]^{-1}, \\
& D= \pm(2 k l)^{-1}[-c k]^{\frac{1}{2}}\left[-k^{2}(1-2 M / R)\right. \\
& \left.+(1-2 M / R)^{-1}\right] \cdot\left[(1-2 M / R)^{-1}\right. \\
& \left.+k^{2}(1-2 M / R)+2 c k\right]^{-1} \text {, }
\end{aligned}
$$

where $C$ and $D$ are defined as before, in Eqs. (115).

If we put

$$
\begin{aligned}
& R=a t^{\prime \prime}+b, \\
& T=h \psi,
\end{aligned}
$$

where $a, b$, and $h$ are constants, we can show after

${ }^{10}$ M. A. Melvin, Phys. Letters 8, 65 (1964). 
some calculation that this is equivalent to Brill space $^{11}$ (electrified NUT space). There are just enough arbitrary constants in this generated solution to reproduce the constants of Brill space. In particular, we note that $c=-1$ yields the Reissner-Nordstrom solution and $c=0$ yields NUT space. ${ }^{12}$ Again we see the complementarity between twist and electromagnetic field.

Equation (123a) is both interesting and puzzling. It shows that in general the origin is shifted by this solution generation. $b$ is usually $\neq 0 \quad(b=0$ only when $k=-c$ ) and is proportional to $M$.

\section{DISCUSSION AND INTERPRETATION OR} RESULTS

An obvious question is: What is the physical and/or mathematical significance of the theorems provable by this method? There is no good answer to this question. One sees in Theorem 2 that the change from old to new metrics amounts to a conformal transformation on the space of the $x^{\alpha}$; but the $x^{k}$ term experiences the inverse of that conformal transformation and may acquire nondiagonal components besides. The relationships of Petrov types, and such physical quantities as stress and shear of the old and new solutions, are also yet to be investigated. At present the method seems primarily to be a way of generalizing known solutions of the equations, while retaining some of the symmetry. It is hoped that this approach may be useful in throwing light on some fundamental properties of the Einstein-Maxwell system of equations. An ideal hope for the approach lies in the possibility of finding ways of combining solutions in arbitrary amounts to get new solutions, analogous to superposition in linear equations.

The method depends heavily on the existence of one Killing vector so that one can define a function $U$. (It should be noted that this Killing vector must be timelike or spacelike; no method exists at present for treating the null Killing-vector case.) One now wonders how closely such theorems as discussed in this paper are tied to the symmetries of the metric. Are there other theorems for metrics with higher symmetries?

One approach, in the case in which there exist two Killing vectors, is to apply the above theorems twiceonce with respect to one variable and once with respect to another variable. Clearly, the metric must be of a rather special form to do this, and the first application of a theorem must not destroy the symmetry necessary for the second. We expect that repeated applications of

\footnotetext{
${ }^{11}$ D. R. Brill, Phys. Rev. 133, B845 (1964).

12 E. Newman, L. Tamburino, and T. Unti, J. Math. Phys. 4, 915 (1963).
}

the same theorem with respect to the same coordinate will merely produce another application of the same family-i.e., the generating transformations of the same type should form a group. This may be checked for the types of transformations in Theorem 2.

Another approach involves setting up a metric of higher symmetry and attempting to prove theorems in ways similar to before. So far, there is only one result available, given here:

Theorem 3: If the following metric is a vacuum solution:

$$
-d s^{2}=\epsilon\left[\eta e^{\alpha+\beta}\left(d x^{l}\right)^{2}+e^{\alpha-\beta}\left(d x^{k}\right)^{2}+\omega_{A B} d x^{A} d x^{B}\right]
$$

and capital Latin letters take $0,1,2,3$, except $k$ and $l$, with $\epsilon= \pm 1, \eta= \pm 1, \partial / \partial x^{k}=\partial / \partial x^{l}=0$, then

$$
\begin{aligned}
-d s^{2}=\epsilon[ & \delta \eta e^{\alpha+\beta-\mu \alpha}\left(d x^{\prime}\right)^{2}+\delta e^{\alpha-\beta+\mu \alpha}\left(d x^{\prime k}\right)^{2} \\
& \left.+a^{2} \exp \left(\frac{1}{2} \mu^{2} \alpha-\mu \beta\right) \omega_{A B} d x^{A} d x^{B}\right]
\end{aligned}
$$

is also a solution, where $\delta= \pm 1$ (used only if $\eta=$ $-1), a$ and $\mu$ are constants, and $x^{\prime k}$ and $x^{\prime}$ are linear combinations of $x^{k}$ and $x^{l}$.

The proof is somewhat involved and will not be given here.

The method is not currently applicable to perfect fluids. Attempts to apply it to fluids have led to the equation of state $P=c^{2} \rho$, which occurs only if the matter has sound speed equal to the speed of light. This occurrence suggests that perhaps these theorems are derivable only for the presence of fields with fundamental velocity $c$. Indeed, one can derive similar theorems for a neutrino energy-momentum tensor. Of course, it is to be hoped-at least for solutiongeneration purposes!-that fluids will eventually be incorporated into the scheme. A small investigation has been made of the derivability of theorems in the Brans-Dicke theory ${ }^{13}$; it appears to be possible, although the mathematics is more involved.

It is anticipated that this method and similar methods will be very useful in general relativity-not only for generating new solutions of the equations, but also for examining more fundamental concepts relating the structure of the equations.

\section{ACKNOWLEDGMENTS}

The author expresses appreciation to Hugo D. Wahlquist and Frank B. Estabrook for conversations about the results of this paper, and to John $H$. Gardner for his continued support of this research.

${ }^{13}$ C. Brans and R. H. Dicke, Phys. Rev. 124, 925 (1961); R. H. Dicke, Phys. Rev. 125, 2163 (1962). 